\title{
Hepatitis $C$ prevalence and risk factors in Georgia, 2015: setting a baseline for elimination
}

Liesl M. Hagan 1*, Ana Kasradze², Stephanie J. Salyer ${ }^{3}$, Amiran Gamkrelidze², Maia Alkhazashvili², Gvantsa Chanturia², Nazibrola Chitadze ${ }^{2}$, Roena Sukhiashvili², Marina Shakhnazarova², Steven Russell ${ }^{3}$, Curtis Blanton ${ }^{3}$, Giorgi Kuchukhidze ${ }^{2}$, Davit Baliashvili², Susan Hariri ${ }^{1}$, Stephen Ko ${ }^{1,4}$, Paata Imnadze ${ }^{2}$, Jan Drobeniuc ${ }^{1}$, Juliette Morgan ${ }^{3,5}$ and Francisco Averhoff ${ }^{1}$

\begin{abstract}
Background: The country of Georgia launched the world's first Hepatitis C Virus (HCV) Elimination Program in 2015 and set a $90 \%$ prevalence reduction goal for 2020. We conducted a nationally representative HCV seroprevalence survey to establish baseline prevalence to measure progress toward elimination over time.

Methods: A cross-sectional seroprevalence survey was conducted in 2015 among adults aged $\geq 18$ years using a stratified, multi-stage cluster design $(n=7000)$. Questionnaire variables included demographic, medical, and behavioral risk characteristics and HCV-related knowledge. Blood specimens were tested for antibodies to HCV (antiHCV) and HCV RNA. Frequencies were computed for HCV prevalence, risk factors, and HCV-related knowledge. Associations between anti-HCV status and potential risk factors were calculated using logistic regression.

Results: National anti-HCV seroprevalence in Georgia was 7.7\% (95\% confidence interval $(\mathrm{Cl})=6.7$, 8.9); HCV RNA prevalence was $5.4 \%(95 \% \mathrm{Cl}=4.6,6.4)$. Testing anti-HCV+ was significantly associated with male sex, unemployment, urban residence, history of injection drug use (IDU), incarceration, blood transfusion, tattoos, frequent dental cleanings, medical injections, dialysis, and multiple lifetime sexual partners. History of IDU (adjusted odds ratio $(A O R)=21.4,95 \% \mathrm{Cl}=12.3,37.4)$ and blood transfusion ( $\mathrm{AOR}=4.5,95 \% \mathrm{Cl}=2.8,7.2)$ were independently, significantly associated with testing anti-HCV+ after controlling for sex, age, urban vs. rural residence, and history of incarceration. Among anti-HCV+ participants, $64.0 \%$ were unaware of their HCV status, and $46.7 \%$ did not report IDU or blood transfusion as a risk factor.
\end{abstract}

Conclusions: Georgia has a high HCV burden, and a majority of infected persons are unaware of their status. Ensuring a safe blood supply, implementing innovative screening strategies beyond a risk-based approach, and intensifying prevention efforts among persons who inject drugs are necessary steps to reach Georgia's HCV elimination goal.

Keywords: Hepatitis C virus, HCV, HCV elimination, Georgia, HCV prevention, Global health security

\footnotetext{
*Correspondence: vqf8@cdc.gov

${ }^{1}$ Division of Viral Hepatitis, Centers for Disease Control and Prevention,

Atlanta, GA, USA

Full list of author information is available at the end of the article
}

(c) The Author(s). 2019 Open Access This article is distributed under the terms of the Creative Commons Attribution 4.0 International License (http://creativecommons.org/licenses/by/4.0/), which permits unrestricted use, distribution, and reproduction in any medium, provided you give appropriate credit to the original author(s) and the source, provide a link to the Creative Commons license, and indicate if changes were made. The Creative Commons Public Domain Dedication waiver (http://creativecommons.org/publicdomain/zero/1.0/) applies to the data made available in this article, unless otherwise stated. 


\section{Background}

Globally, there are an estimated 71 million people living with hepatitis $\mathrm{C}$ virus (HCV) infection and 411,000 HCV-attributable deaths annually [1]. HCV is bloodborne and transmitted most often through unsterile medical equipment, infected blood and tissue used for medical procedures, and shared drug injection equipment. HCV infection often progresses asymptomatically for 20-30 years, and most HCV-related deaths result from liver cirrhosis or hepatocellular carcinoma decades after the incident $\mathrm{HCV}$ infection [2-6]. HCV accounts for an estimated $27 \%$ of cirrhosis and $25 \%$ of hepatocellular carcinoma cases worldwide [7].

Georgia is an Eastern European, middle-income country with 3.7 million residents [8]. A 2002 survey in the capital city of Tbilisi found that $6.7 \%$ of the general population and $70.4 \%$ of persons who inject drugs had antibodies to $\mathrm{HCV}$ (anti-HCV, evidence of past or current $\mathrm{HCV}$ infection) [9], suggesting that $\mathrm{HCV}$ prevalence in Georgia could be among the highest globally. In 2015, Georgia launched the world's first HCV elimination program, aiming to provide universal access to curative, direct-acting antiviral (DAA) treatment at no cost to patients, and to implement nationwide prevention measures to curb transmission [10]. Existing prevalence data have been instrumental in engaging the government's strong support to combat the country's HCV burden, but are outdated and not nationally representative. Data documenting updated nationwide HCV prevalence and risk factors for infection are necessary to effectively plan treatment and prevention services supporting Georgia's HCV elimination goals, and to establish a baseline to track progress toward elimination over time.

This paper presents the results of the first nationally representative $\mathrm{HCV}$ seroprevalence survey in Georgia, conducted in 2015 by Georgia's National Center for Disease Control and Public Health (NCDC) in collaboration with the United States Centers for Disease Control and Prevention (CDC). The Georgian government is using these results to plan and implement HCV surveillance, education, prevention, screening, care, and treatment efforts. A follow-up survey is planned to assess the impact of interventions designed to achieve $\mathrm{HCV}$ elimination. In addition, planning and conducting this national serosurvey provided an important opportunity to strengthen the public health capacity in Georgia and thereby enhance global health security.

\section{Methods}

\section{Sample design}

A cross-sectional, nationally representative seroprevalence survey was conducted in Georgia from May-August 2015 among adults aged $\geq 18$ years using a stratified, multi-stage cluster design. A sample size of 7000 was calculated based on estimated $6.7 \%$ anti-HCV seroprevalence [9], a design effect of 2 , and an anticipated $70 \%$ response rate. The sample was designed to yield a nationwide $\mathrm{HCV}$ prevalence estimate, independent prevalence estimates in six pre-selected major cities, and in urban vs. rural areas overall. Region-level estimates were also calculated where sample size was sufficient.

The country was divided into 16 mutually exclusive sampling strata (six major cities and ten regions). Strata defined by a region contained both urban and rural areas, but excluded any of the six major cities that lie within the region's boundaries. The occupied territories of Abkhazia and South Ossetia were excluded.

The sampling frame was a full list of Georgia's 9503 census tracts, provided by Georgia's National Statistics Office (GeoStat). These census tracts served as the primary sampling units (clusters) within each stratum. Equal size tracts were assumed since a size measure was not available during sample selection. To reach a sample size of 7000, 280 clusters were selected across the 16 strata, 25 households were selected within each cluster, and one participant was selected from each household. The six major cities were oversampled (120 clusters) to increase precision of point estimates. The remaining 160 clusters were allocated to the ten regions proportionally based on their population size. The specific clusters sampled within each stratum were randomly selected from the list provided by GeoStat.

Within each selected cluster, 25 households were systematically selected using an algorithm based on the cluster's total number of year-round households. Within each household, the Kish method was applied to randomly select one adult for participation [11]. Household members aged $\geq 18$ years who had spent the previous night in the house were eligible; temporary guests and household members living outside the home were excluded. If the selected individual was unavailable, two revisit attempts were made; no replacement participants were selected if the individual was unavailable after revisits or refused participation.

\section{Data collection}

Interviewers trained and supervised by NCDC and CDC epidemiologists administered a structured questionnaire to participants who provided informed consent. The questionnaire was given verbally in participants' preferred language (Kartuli/Georgian, Russian, Armenian, or Azeri) and included demographics, medical history, lifestyle/behavioral history, and knowledge of HCV. Data were entered into hand-held electronic devices in real time and uploaded to a secure database. Survey questions were vetted by local staff to ensure cultural appropriateness and suitability for laypersons with a primary 
school education. Field and laboratory procedures, questionnaires, and informed consent forms were piloted in rural and urban areas.

Nurse-phlebotomists collected $10 \mathrm{~mL}$ blood specimens from consenting participants. Specimens were centrifuged in the field, transported to public health laboratories for processing and testing, and stored at the Georgian National Reference Laboratory in Tbilisi. Each participant's specimen and questionnaire data were linked using a unique barcode. Personal identifying information was obtained strictly to report laboratory test results to participants, and was removed before epidemiologic analysis.

\section{Laboratory methods}

Anti-HCV and HCV RNA testing were performed in Georgian public health laboratories. CDC laboratory staff monitored protocols and processes for quality assurance/quality control. All specimens were tested for anti-HCV by enzyme-immunoassay (HCV Ab v4.0 EIA $I V D$, Dia.Pro. Diagnostic Bioprobes Srl, Italy). Anti-HCV-positive specimens were tested for $\mathrm{HCV}$ RNA (Sacace $^{\mathrm{Tm}}$ HCV Real-TM Qual, Sacace Biotechnologies Srl, Italy). Anti-HCV-positive/RNA-negative specimens underwent confirmatory anti-HCV testing using a third generation line immunoassay (INNO-LIA $^{\text {тм }}$ HCV Score, IVD, Innogenetics N.V., Belgium); specimens that tested positive or indeterminate for anti-HCV in confirmatory testing were re-tested for HCV RNA in the CDC Division of Viral Hepatitis Assay Development and Diagnostic Reference Laboratory in Atlanta, Georgia, USA using a highly sensitive, FDA-licensed assay (COBAS Ampliprep/COBAS Taqman $^{\circ}$ CAP/CTM v2.0, IVD, Roche, Indianapolis, IN, USA); specimens testing HCV RNA negative in the $\mathrm{CDC}$ laboratory were re-tested for anti-HCV using the FDA-licensed VITROS Immunodiagnostic System (aHCV, IVD, Ortho Clinical Diagnostics, Raritan, NJ, USA) to identify false positives. All specimens were tested for hepatitis B virus and human immunodeficiency virus; results are not reported in this manuscript.

Laboratory test results were reported securely to participants via the Georgian Post; to receive the mailing, participants were required to present a national identification card matching the name of the addressee. Participants with a positive HCV RNA test received written instructions for accessing Georgia's national HCV treatment program in the same mailing.

\section{Statistical methods}

Statistical analyses were conducted using SAS 9.3 (Cary, North Carolina, USA). Data were weighted based on probability of selection at cluster, household, and individual levels, and adjusted to represent Georgia's national population by sex, age, and geographic distribution using 2014 census data. Analyses used complex survey procedures accounting for stratification, clustering, and unequal sample weights. Variance was calculated using Taylor series linearization.

Anti-HCV prevalence was calculated by demographic characteristics and potential $\mathrm{HCV}$ risk factors; weighted prevalence estimates and 95\% confidence intervals (CI) are presented. Bivariate associations between anti-HCV positivity and demographic and risk factor characteristics were examined using Rao-Scott chi-square tests; associations were considered significant when $p<.05$. An unconditional logistic regression model was utilized to explore the relationship between anti-HCV positivity and multiple risk factors that were significantly associated with anti-HCV status in bivariate analyses. Backward elimination was used to reduce the full model; variables were retained if the Wald $\mathrm{F}$ test $\mathrm{p}<.05$. Variables without significant, independent associations with anti-HCV positivity were retained as confounders if they changed parameter estimates for other significant predictor variables in the main effects model by $\geq 10 \%$. All potential pairwise interactions in the final model were examined and considered significant if the Wald $\mathrm{F}$ test $\mathrm{p}<.05$. The final model was assessed for multicollinearity. Odds ratios and 95\% CI are presented. Weighted percentages and $95 \% \mathrm{CI}$ were computed for $\mathrm{HCV}$ knowledge variables. Unweighted percentages were computed for $\mathrm{HCV}$ treatment history variables.

This survey was determined to be a routine public health activity for public health surveillance by CDC's Human Subjects Research Office and therefore judged to not involve human subjects research.

\section{Results}

Of 7000 adults selected, 6296 (89.9\%) consented to participate, and 6014 (85.9\%) provided both questionnaire responses and a blood specimen. Response rates exceeded $70 \%$ in all strata. Three specimens were hemolyzed during processing, and one returned inconclusive anti-HCV results. Demographic analyses include all 6296 participants; HCV-specific analyses include the 6010 participants who provided both questionnaire responses and a blood specimen yielding interpretable serologic test results.

\section{Participant demographics and exposures}

Participants' median age was 45 years; $53.8 \%$ were female, and $56.7 \%$ lived in urban areas (Table 1). 90.9\% had completed secondary school or higher, and $19.5 \%$ were unemployed. Approximately two-thirds (64.0\%) reported an annual household income less than the national average (12,268 Georgian Lari/\$5254 US dollars) [12] . 
Table 1 Demographic characteristics and reported exposures among survey participants, Georgia HCV serosurvey, 2015

\begin{tabular}{|c|c|c|}
\hline Characteristic & $n$ & Weighted \% (95\% Cl) \\
\hline Total Sample & 6296 & 100.0 \\
\hline \multicolumn{3}{|l|}{ Sex } \\
\hline Female & 3868 & $53.8(52.0,55.5)$ \\
\hline Male & 2428 & $46.2(44.5,48.0)$ \\
\hline Missing & 0 & \\
\hline \multicolumn{3}{|l|}{ Age } \\
\hline $18-29$ & 1115 & $19.4(18.2,20.7)$ \\
\hline $30-39$ & 1177 & $19.4(17.9,20.9)$ \\
\hline $40-49$ & 1070 & $18.6(17.2,20.0)$ \\
\hline $50-59$ & 1140 & $16.5(15.4,17.7)$ \\
\hline$\geq 60$ & 1790 & $26.1(24.5,27.8)$ \\
\hline Missing & 4 & \\
\hline \multicolumn{3}{|l|}{ Geography } \\
\hline Urban & 3350 & $56.7(52.7,60.6)$ \\
\hline Rural & 2946 & $43.3(39.4,47.3)$ \\
\hline Missing & 0 & \\
\hline \multicolumn{3}{|l|}{ Employment status } \\
\hline Employed & 2120 & $37.8(35.6,39.9)$ \\
\hline Student & 172 & $3.6(2.9,4.4)$ \\
\hline Homemaker & 1483 & $19.1(17.7,20.6)$ \\
\hline Retired & 1405 & $20.0(18.7,21.5)$ \\
\hline Unemployed (able to work) & 1110 & $19.5(18.0,21.1)$ \\
\hline Missing & 6 & \\
\hline \multicolumn{3}{|l|}{ Highest level of education completed } \\
\hline Completed less than elementary/primary school & 43 & $0.7(0.5,1.1)$ \\
\hline Completed elementary/primary school & 612 & $8.5(7.3,9.8)$ \\
\hline Completed secondary school & 2567 & $40.2(38.1,42.3)$ \\
\hline Completed professional/technical school & 1157 & $16.6(15.3,18.0)$ \\
\hline Completed university/college or higher & 1912 & $34.0(31.6,36.4)$ \\
\hline Missing & 5 & \\
\hline \multicolumn{3}{|l|}{ Yearly household income } \\
\hline$\leq 6000$ GEL/year ( $\leq 4400$ USD) & 2867 & $45.6(43.0,48.3)$ \\
\hline 6001-12,000 GEL/year (4400-6800 USD) & 953 & $18.5(16.8,20.3)$ \\
\hline $12,001-24,000$ GEL/year (6800-13,600 USD) & 724 & $12.6(11.3,13.9)$ \\
\hline$>24,000$ GEL/year (> 13,600 USD) & 1339 & $23.3(21.1,25.8)$ \\
\hline Missing & 413 & \\
\hline \multicolumn{3}{|l|}{ Ever injected drugs } \\
\hline Yes & 208 & $4.2(3.5,5.2)$ \\
\hline No & 6042 & $95.8(94.8,96.5)$ \\
\hline Missing & 46 & \\
\hline \multicolumn{3}{|l|}{ Ever incarcerated } \\
\hline Yes & 240 & $4.6(3.8,5.7)$ \\
\hline No & 6037 & $95.4(94.3,96.2)$ \\
\hline Missing & 19 & \\
\hline
\end{tabular}


Table 1 Demographic characteristics and reported exposures among survey participants, Georgia HCV serosurvey, 2015 (Continued)

\begin{tabular}{|c|c|c|}
\hline Characteristic & $\mathrm{n}$ & Weighted \% (95\% Cl) \\
\hline \multicolumn{3}{|l|}{ Have any tattoos } \\
\hline Yes & 639 & $12.2(10.9,13.7)$ \\
\hline No & 5645 & $87.8(86.3,89.1)$ \\
\hline Missing & 12 & \\
\hline \multicolumn{3}{|c|}{ Ever received a blood transfusion } \\
\hline Yes & 459 & $7.0(6.1,7.9)$ \\
\hline No & 5828 & $93.0(92.1,93.9)$ \\
\hline Missing & 9 & \\
\hline \multicolumn{3}{|c|}{ Ever received kidney dialysis } \\
\hline Yes & 17 & $0.3(0.2,0.6)$ \\
\hline No & 6255 & $99.7(99.4,99.8)$ \\
\hline Missing & 24 & \\
\hline \multicolumn{3}{|c|}{ Number of medical injections received in last 6 months } \\
\hline 0 & 3857 & $62.8(60.7,64.8)$ \\
\hline 1 & 557 & $9.5(8.4,10.7)$ \\
\hline$>1$ & 1701 & $27.8(26.0,29.6)$ \\
\hline Missing & 181 & \\
\hline \multicolumn{3}{|c|}{ Frequency of dental cleanings } \\
\hline Twice/year & 199 & $4.4(3.6,5.3)$ \\
\hline Once/year & 491 & $9.0(7.8,10.2)$ \\
\hline Less than once/year & 1170 & $20.3(18.5,22.3)$ \\
\hline Never & 4370 & $66.3(64.0,68.5)$ \\
\hline Missing & 66 & \\
\hline \multicolumn{3}{|c|}{ Number of lifetime sexual partners } \\
\hline $0-2$ & 4232 & $75.0(73.1,76.8)$ \\
\hline$>2$ & 1026 & $25.0(23.2,26.9)$ \\
\hline Missing & 1038 & \\
\hline \multicolumn{3}{|c|}{ Men who have sex with men (MSM) } \\
\hline Yes & 0 & 0 \\
\hline No & 2185 & 90.0 \\
\hline Missing & 243 & 10.0 \\
\hline
\end{tabular}

When asked about risk factors for HCV infection, $4.2 \%$ reported a history of injection drug use (IDU), $7.0 \%$ reported receiving a blood transfusion, and $<1 \%$ reported receiving dialysis; $4.6 \%$ reported a history of incarceration, and $12.2 \%$ reported having at least one tattoo. None identified as men who have sex with men (MSM), and 25.0\% reported having > 2 lifetime sexual partners.

\section{$\mathrm{HCV}$ prevalence}

Of the 6010 participants providing a usable blood specimen, $433 \quad(7.7,95 \% \quad \mathrm{CI}=6.7,8.9) \quad$ tested anti-HCV positive, and $311(5.4,95 \% \mathrm{CI}=4.6,6.4)$ tested HCV RNA positive (indicating chronic infection). Anti-HCV prevalence was higher in urban vs. rural areas $(9.5 \%$ vs. $5.4 \%, p<0.0001)$ (Table 2$)$; the highest regional prevalence was in Samegrelo-Zemo Svaneti region in northwest Georgia (10.9\%), particularly in the city of Zugdidi (14.0\%) (Fig. 1). Anti-HCV prevalence was approximately three times higher among men vs. women $(12.1 \%$ vs. $3.8 \%, \mathrm{p}<$ 0.0001 ) and varied by age (Table 2); among men, prevalence peaked at $22.7 \%$ in the $40-49$ age group, while it increased steadily with age among women to a maximum of $5.4 \%$ among those $\geq 60$ years of age (Fig. 2). 
Table 2 Anti-HCV prevalence by demographic and exposure subgroup in unadjusted and adjusted models, Georgia HCV serosurvey, 2015

\begin{tabular}{|c|c|c|c|c|c|c|c|}
\hline \multirow[t]{2}{*}{ Characteristic } & \multirow[b]{2}{*}{ Total $n$} & \multicolumn{2}{|c|}{ Anti-HCV Prevalence } & \multicolumn{2}{|c|}{ Unadjusted Models } & \multicolumn{2}{|c|}{ Final Adjusted Model } \\
\hline & & $\mathrm{n}$ & Weighted \% (95\% Cl) & Crude OR (95\% Cl) & $p$-value & Adjusted OR (95\% Cl) & p-value \\
\hline \multicolumn{8}{|l|}{ Demographics } \\
\hline \multicolumn{8}{|l|}{ Sex } \\
\hline Female & 3671 & 145 & $3.8(3.0,4.9)$ & 1 & & & \\
\hline Male & 2339 & 288 & $12.1(10.2,14.3)$ & $3.5(2.5,4.8)$ & $<0.0001$ & & \\
\hline Missing & 0 & & & & & & \\
\hline \multicolumn{8}{|l|}{ Age } \\
\hline $18-29$ & 1063 & 23 & $2.4(1.5,4.0)$ & 1 & & & \\
\hline $30-39$ & 1140 & 94 & $8.8(6.8,11.3)$ & $3.9(2.2,6.8)$ & $<0.0001$ & & \\
\hline $40-49$ & 1026 & 128 & $14.0(11.1,17.6)$ & $6.5(3.9,11.1)$ & $<0.0001$ & & \\
\hline $50-59$ & 1096 & 79 & $7.0(5.2,9.5)$ & $3.0(1.6,5.8)$ & 0.0006 & & \\
\hline $60+$ & 1681 & 109 & $6.7(5.0,9.0)$ & $2.9(1.6,5.4)$ & 0.0007 & & \\
\hline Missing & 4 & & & & & & \\
\hline \multicolumn{8}{|l|}{ Geography } \\
\hline Urban & 3155 & 290 & $9.5(8.0,11.4)$ & $1.8(1.4,2.5)$ & $<0.0001$ & & \\
\hline Rural & 2855 & 143 & $5.4(4.4,6.6)$ & 1 & & & \\
\hline Missing & 0 & & & & & & \\
\hline \multicolumn{8}{|l|}{ Employment Status } \\
\hline Employed/student/ & 4939 & 286 & $5.9(5.0,7.1)$ & 1 & & & \\
\hline \multicolumn{8}{|l|}{ homemaker/unpaid } \\
\hline \multicolumn{8}{|l|}{ worker/retired } \\
\hline Unemployed* & 1065 & 147 & $15.0(12.3,18.1)$ & $2.8(2.1,3.7)$ & $<0.0001$ & & \\
\hline Missing & 6 & & & & & & \\
\hline \multicolumn{8}{|l|}{ Exposures } \\
\hline \multicolumn{8}{|l|}{ Ever injected drugs } \\
\hline Yes & 205 & 150 & $66.5(56.0,75.6)$ & $37.6(23.5,60.0)$ & $<0.0001$ & $21.4(12.3,37.4)$ & $<0.0001$ \\
\hline No & 5762 & 283 & $5.0(4.3,5.9)$ & 1 & & & \\
\hline Missing & 43 & & & & & & \\
\hline \multicolumn{8}{|l|}{ Ever incarcerated } \\
\hline Yes & 236 & 98 & $42.0(32.8,51.7)$ & $11.3(7.5,17.1)$ & $<0.0001$ & & \\
\hline No & 5757 & 335 & $6.0(5.1,7.0)$ & 1 & & & \\
\hline Missing & 17 & & & & & & \\
\hline \multicolumn{8}{|l|}{ Have any tattoos } \\
\hline Yes & 626 & 104 & $16.2(12.2,21.1)$ & $2.8(1.9,4.0)$ & $<0.0001$ & & \\
\hline No & 5372 & 329 & $6.5(5.5,7.6)$ & 1 & & & \\
\hline Missing & 12 & & & & & & \\
\hline \multicolumn{8}{|c|}{ Number of medical injections in last 6 months } \\
\hline 0 & 3656 & 233 & $6.7(5.6,7.9)$ & 1 & & & \\
\hline 1 & 541 & 40 & $6.6(4.3,10.2)$ & $0.99(0.60,1.65)$ & 0.98 & & \\
\hline$>1$ & 1648 & 144 & $9.5(7.5,12.1)$ & $1.48(1.10,1.99)$ & 0.01 & & \\
\hline Missing & 165 & & & & & & \\
\hline
\end{tabular}


Table 2 Anti-HCV prevalence by demographic and exposure subgroup in unadjusted and adjusted models, Georgia HCV serosurvey, 2015 (Continued)

\begin{tabular}{|c|c|c|c|c|c|c|c|}
\hline \multirow[t]{2}{*}{ Characteristic } & \multirow[b]{2}{*}{ Total n } & \multicolumn{2}{|c|}{ Anti-HCV Prevalence } & \multicolumn{2}{|c|}{ Unadjusted Models } & \multicolumn{2}{|c|}{ Final Adjusted Model } \\
\hline & & $\mathrm{n}$ & Weighted \% (95\% Cl) & Crude OR (95\% Cl) & $p$-value & Adjusted OR (95\% Cl) & $p$-value \\
\hline \multicolumn{8}{|c|}{ Ever received a blood transfusion } \\
\hline Yes & 447 & 69 & $21.4(15.6,28.5)$ & $3.8(2.6,5.5)$ & $<0.0001$ & $4.5(2.8,7.2)$ & $<0.0001$ \\
\hline No & 5554 & 364 & $6.7(5.8,7.7)$ & 1 & & & \\
\hline Missing & 9 & & & & & & \\
\hline \multicolumn{8}{|c|}{ Received a blood transfusion before or after 1997} \\
\hline Before 1997 & 225 & 36 & $25.3(16.2,37.3)$ & $1.6(0.7,3.7)$ & 0.27 & & \\
\hline In or after 1997 & 222 & 33 & $17.4(10.7,27.1)$ & 1 & & & \\
\hline \multicolumn{8}{|c|}{ Ever received kidney dialysis } \\
\hline Yes & 17 & 3 & $27.6(7.9,62.9)$ & $4.6(1.0,20.4)$ & 0.04 & & \\
\hline No & 5972 & 430 & $7.7(6.7,8.8)$ & 1 & & & \\
\hline Missing & 21 & & & & & & \\
\hline \multicolumn{8}{|c|}{ Frequency of dental cleanings } \\
\hline Twice/year & 193 & 15 & $15.0(8.1,26.2)$ & $2.1(1.1,4.4)$ & 0.04 & & \\
\hline Once/year & 478 & 27 & $6.7(3.9,11.5)$ & $0.9(0.5,1.6)$ & 0.66 & & \\
\hline Less than once/year & 1108 & 84 & $6.9(5.0,9.4)$ & $0.9(0.6,1.3)$ & 0.58 & & \\
\hline Never & 4173 & 304 & $7.6(6.5,8.8)$ & 1 & & & \\
\hline Missing & 58 & & & & & & \\
\hline \multicolumn{8}{|c|}{ Number of lifetime sexual partners } \\
\hline $0-2$ & 4020 & 157 & $3.8(3.0,4.7)$ & 1 & & & \\
\hline$>2$ & 991 & 129 & $11.9(9.1,15.4)$ & $3.4(2.4,5.0)$ & $<0.0001$ & & \\
\hline Missing & 999 & & & & & & \\
\hline
\end{tabular}

Note: Anti-HCV related analyses include only participants who submitted both questionnaire data and a usable blood specimen $(n=6010)$

*Unemployed includes those able or unable to work

\section{Factors associated with HCV infection}

In bivariate analysis, anti-HCV positivity was significantly associated with male sex, unemployment, and urban residence, as well as history of IDU, incarceration, blood transfusion, tattoos, frequent dental cleanings, medical injections, dialysis, and having multiple lifetime sexual partners (Table 2). Among participants who reported a history of blood transfusion, no significant difference in anti-HCV prevalence was detected between those who reported receiving a transfusion before vs. in/ after 1997 (when Georgia began testing donated blood for HCV) (Table 2). Other medical and community exposures including hospitalization, surgery, body piercings, and manicures/pedicures were not significantly associated with anti-HCV positivity (data not shown).

In the adjusted model, history of IDU (adjusted odds ratio $(\mathrm{AOR})=21.4,95 \% \mathrm{CI}=12.3,37.4)$ and receipt of a blood transfusion at any date $(\mathrm{AOR}=4.5,95 \% \mathrm{CI}=2.8$, 7.2) were the only risk factors that were significantly, independently associated with anti-HCV positivity, controlling for sex, age, urban vs. rural residence, and history of incarceration (Table 2). [Note: A dichotomous blood transfusion variable (ever vs. never received transfusion) was used in the multivariate model.] There were no significant interactions in the final model.

Of the 433 anti-HCV positive participants, $38.2 \%$ reported IDU, and $19.7 \%$ reported receiving a blood transfusion. Nearly half of anti-HCV positive participants (46.7\%) did not report either of these risk factors. Overall, $66.5 \%$ of anti-HCV positive participants were male, and $43.4 \%$ were $\geq$ age 50 . The sex and age breakdown was similar among anti-HCV positive participants reporting a blood transfusion $(63.2 \%$ male and $55.7 \% \geq$ age 50) and among anti-HCV positive participants who did not report either IDU or history of blood transfusion (60.6\% male and $46.2 \% \geq$ age 50 ). Anti-HCV positive participants reporting IDU were mostly male (98.3\%) and concentrated in the 30-49 age range (70.0\%), with $16.3 \% \geq$ age 50 .

\section{HCV diagnosis and treatment}

Among the 433 participants who tested anti-HCV positive, 156 (36.0\%) already knew their HCV status prior to the survey. Awareness of $\mathrm{HCV}$ status was more likely 


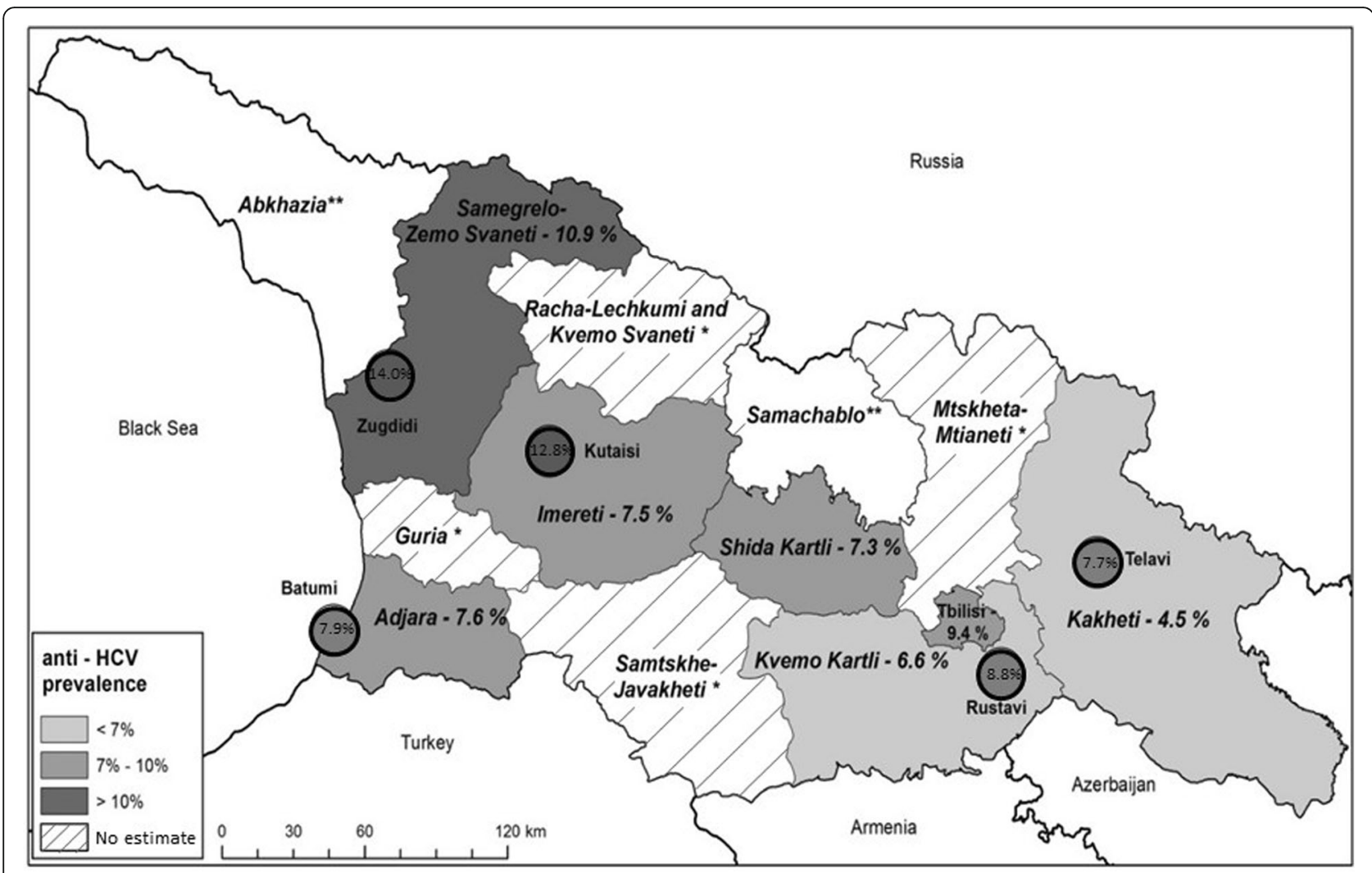

Fig. 1 Anti-HCV prevalence in major cities and regions of Georgia. The highest regional anti-HCV prevalence was found in Samegrelo-Zemo Svaneti region in northwest Georgia (10.9\%), particularly in the city of Zugdidi (14.0\%, nearly double the national prevalence of $7.7 \%)$. In general, anti-HCV prevalence was higher in cities than in the surrounding rural areas. [Notes: *Anti-HCV prevalence estimates were not calculated for Guria region, Mtskheta-Mtianeti region, Racha-Lechkumi/Kvemo Svaneti region, or Samtskhe-Javakheti region due to insufficient sample size. **The occupied territories of Abkhazia and Samachablo (South Ossetia) were not included in the survey]

among anti-HCV positive participants reporting IDU compared to those not reporting IDU (55.3\% vs. $28.5 \%$, $p=0.0002)$. Among participants aware of their HCV infection, 50 (32.1\%) reported initiating treatment prior to the survey, $32(64.0 \%)$ of those who reported initiating treatment reported completing it, and 6 (18.8\%) of those who reported completing treatment reported being cured (Fig. 3). A cross-check of self-reports against laboratory test results revealed that 14 participants reporting treatment completion tested $\mathrm{HCV}$ RNA negative

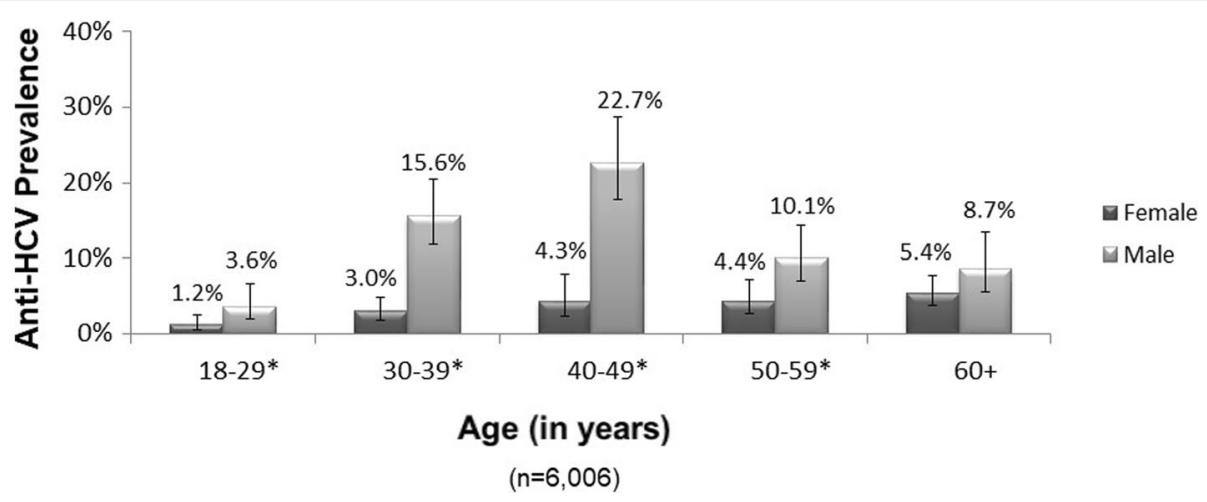

Fig. 2 Anti-HCV prevalence by age and sex. Anti-HCV prevalence was approximately three times higher among men vs. women overall (12.1\% vs. $3.8 \%$ ) and varied by age; among men, prevalence peaked at $22.7 \%$ in the $40-49$ age group, while it increased steadily with age among women to a maximum of $5.4 \%$ among those $\geq 60$ years of age. [Note: * Differences in anti-HCV prevalence between male and female respondents were statistically significant in asterisked categories using Rao-Scott Chi-square tests $(p<0.05)]$ 


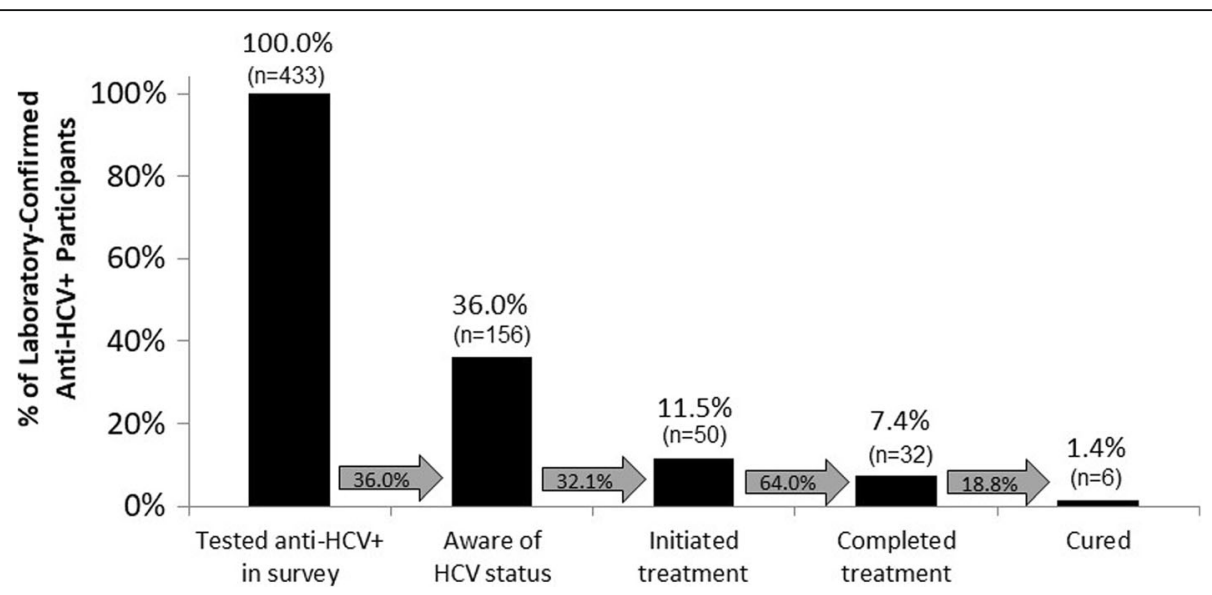

Fig. 3 Self-reported cascade of HCV care among laboratory-confirmed anti-HCV positive participants. Among the 433 survey participants who tested anti-HCV positive, 156 (36.0\%) already knew their HCV status prior to the survey. Among participants aware of their HCV infection, 50 (32.1\%) reported initiating treatment prior to the survey, 32 (64.0\%) of those who began treatment reported completing it, and 6 (18.8\%) of those who completed treatment reported being cured

(more than twice the number who reported being cured); however only three of the six who reported a cure actually tested HCV RNA negative.

Among anti-HCV positive participants aware of their infection and reporting no treatment, reasons cited for non-treatment included lack of treatment availability (56.6\%), high cost (33.0\%), and anticipated side effects (12.3\%).

\section{HCV-related knowledge}

A majority of participants (56.1\%) were aware that $\mathrm{HCV}$ can be transmitted through exposure to infected blood; when asked about specific transmission modes, 52.3\% identified sharing needles/syringes, $43.6 \%$ identified sharing household objects that have had contact with blood, and $31.9 \%$ identified sexual contact as possible $\mathrm{HCV}$ transmission modes. More than half of participants (57.2\%) were aware that $\mathrm{HCV}$ can be asymptomatic, and $70.5 \%$ knew that HCV is treatable. HCV-related knowledge was higher among participants who were anti-HCV positive, and highest specifically among
anti-HCV positive participants reporting a history of IDU (Table 3).

When asked what sources they trust for information about their health, $35.8 \%$ of participants identified doctors and other healthcare workers, and $34.0 \%$ identified television. Other information sources including the internet, family/friends, medical literature, newspapers, radio, brochures/fliers, pharmacists, and billboards, were each cited as trustworthy by fewer than 15\% of participants (data not shown). Participants were able to select multiple responses to this question.

\section{Discussion}

HCV elimination has garnered increasing international support since the development of curative HCV drugs in recent years, resulting in the World Health Organization's worldwide HCV elimination plan, the European Union HCV Policy Summit commitment to elimination, and individual elimination programs in Georgia, Australia, Iceland, the Cherokee Nation in the United States, and other areas [13-17]. Georgia was the first

Table 3 HCV-related knowledge by anti-HCV status and reported IDU history, Georgia HCV serosurvey, 2015

\begin{tabular}{|c|c|c|c|c|c|c|}
\hline & \multicolumn{2}{|c|}{ All participants } & \multicolumn{2}{|c|}{ Anti-HCV+ participants } & \multicolumn{2}{|c|}{ Anti-HCV+ participants reporting IDU } \\
\hline & $n$ & Weighted \% (95\% Cl) & $n$ & Weighted \% (95\% Cl) & $n$ & Weighted \% (95\% Cl) \\
\hline Aware that HCV can be asymptomatic & 2458 & $57.2(54.9,59.4)$ & 260 & $76.4(69.9,81.9)$ & 120 & $83.7(74.2,90.2)$ \\
\hline Aware that HCV can be treated & 3041 & $70.5(68.5,72.5)$ & 287 & $83.6(77.5,88.3)$ & 130 & $89.0(80.1,94.2)$ \\
\hline \multicolumn{7}{|l|}{ HCV can be transmitted by } \\
\hline Blood & 3295 & $56.1(53.9,58.3)$ & 295 & $71.1(64.8,76.8)$ & 136 & $89.0(80.2,94.1)$ \\
\hline Sharing needles or syringes & 3056 & $52.3(50.0,54.6)$ & 278 & $67.1(60.4,73.2)$ & 128 & $87.2(78.8,92.6)$ \\
\hline Sharing household objects like & 2582 & $43.6(41.1,46.1)$ & 249 & $58.6(51.7,65.1)$ & 114 & $73.4(62.4,82.1)$ \\
\hline \multicolumn{7}{|l|}{ razors or toothbrushes } \\
\hline Sexual contact & 1875 & $31.9(30.1,33.7)$ & 165 & $41.4(35.0,48.1)$ & 83 & $57.5(46.3,67.9)$ \\
\hline
\end{tabular}


country to undertake HCV elimination and has set ambitious targets including a 90\% reduction in chronic HCV prevalence by 2020 [10, 18].

This survey confirms that Georgia has a high burden of $\mathrm{HCV}$ infection and identifies risk factors that will be essential to address in Georgia's HCV elimination strategy. Applying the 5.4\% HCV RNA prevalence found in this survey to Georgia's adult population of 2.78 million results in an estimated 150,340 (95\% CI $=128,060$, $173,060)$ people aged $\geq 18$ years living with chronic $\mathrm{HCV}$ infection. Because this sample did not include incarcerated or homeless persons, groups known to have high HCV prevalence [19-22], this survey likely underestimates the true HCV burden. Two risk factors measured in this survey were significant, independent predictors of anti-HCV positivity: reported history of IDU and reported receipt of a blood transfusion. However, half of anti-HCV positive participants reported neither exposure, illustrating that screening based on reported risk factors alone will be insufficient to identify most chronically infected persons and eliminate HCV.

Communication about HCV transmission modes and disease course will be important components of efforts to increase screening. Half of all participants were unaware that they could have an $\mathrm{HCV}$ infection without experiencing any symptoms, and half were unaware that $\mathrm{HCV}$ is transmitted through exposure to infected blood. HCV-related knowledge was highest among participants reporting a history of IDU, possibly due to familiarity with the risks of injecting drugs. Although media coverage of the $\mathrm{HCV}$ elimination program within Georgia has likely increased the general public's knowledge about HCV since this survey, these findings highlight the need to further intensify public education efforts to drive screening, particularly in groups less familiar with $\mathrm{HCV}$ transmission risks such as injecting drugs. However, identifying effective messaging and modes of communication could be challenging, given that only one-third of participants expressed trust in healthcare professionals as sources of health-related information, and even fewer reported trust in other sources including friends, family, radio, television, or the internet.

History of IDU was the strongest predictor of HCV infection in this survey and was reported by $38.2 \%$ of anti-HCV positive participants. IDU was most common among men, likely driving the three-fold difference in anti-HCV prevalence between men vs. women. In particular, men ages $40-49$ years had the highest prevalence of both reported IDU (17.4\%, data not shown) and anti-HCV (22.7\%). (This cohort came of age during a drug trafficking and IDU epidemic in Georgia during the 1990s/early 2000s following the collapse of the former Soviet Union [23]). However, injecting behavior poses an important challenge for $\mathrm{HCV}$ elimination regardless of the age of persons injecting, and those actively injecting drugs will be a key target to curb transmission. Increasing access to harm reduction programs, including needle and syringe programs and medication for opioid use disorder, will be essential. In addition, a follow-up study among persons actively injecting drugs would further clarify HCV prevalence and risk behaviors in this sub-group to guide prevention efforts.

History of a blood transfusion also emerged as an independent risk factor for $\mathrm{HCV}$ infection and was reported by $20 \%$ of anti-HCV positive participants. Although Georgia began testing its donated blood supply for HCV in 1997, there was no detectable difference in anti-HCV prevalence between participants who received a transfusion before vs. after the blood testing program began. To halt HCV transmission and support elimination, it is imperative that Georgia evaluate and improve its blood safety program.

Nearly half of anti-HCV positive participants reported neither IDU nor blood transfusion. Possible explanations include underreporting of risk factors due to stigma, legal concerns, and poor recall, as well as HCV transmission through exposures not identified as potential risk factors in this survey. Suboptimal infection control during healthcare and dental procedures has been hypothesized as an $\mathrm{HCV}$ transmission risk in Georgia due to privatization and regulatory challenges in these sectors following the dissolution of the former Soviet Union. However, the cross-sectional nature of this survey and the near-universal utilization of dental and healthcare services make risk association difficult to detect from these exposures. Nonetheless, these data indicate that nearly half of $\mathrm{HCV}$-infected persons in Georgia could be unaware of their risk history or unwilling to report it. Thus, screening strategies beyond a risk-based approach will be necessary for Georgia to identify enough infected persons to reach its elimination targets. In addition, further investigation is warranted to better understand potential HCV transmission risks in Georgia aside from IDU and blood transfusions, as well as differences in risk factors by sex.

Over $60 \%$ of participants with evidence of HCV infection learned about their status for the first time through participation in this survey. Among those already aware of their HCV infection, approximately one-third reported prior treatment; most would have been treated with interferon-based regimens, which were the only $\mathrm{HCV}$ treatment options available in Georgia prior to the launch of the national elimination program, and were cost-prohibitive for most Georgians. By offering DAA-based treatment to patients at no cost, Georgia's HCV elimination program has addressed the primary treatment barriers cited by survey participants - expense, availability, and anticipated side effects. From the beginning of the elimination program in April 2015 through December 2016, 27,595 persons 
initiated treatment, and efforts are ongoing to continue to improve access for those who are aware of their HCV infection $[18,24]$. With treatment infrastructure now in place, the greatest opportunity to boost progress toward $\mathrm{HCV}$ elimination lies in screening and diagnosing more infected individuals.

This survey has several limitations. Its cross-sectional design limits the ability to draw causal associations between possible exposures and $\mathrm{HCV}$, a chronic infection that could have been acquired at any time before the survey. Further, the necessary reliance on self-reported risk factor data could result in information bias that is unmeasurable. The fact that IDU is illegal in Georgia and is the leading reason for incarceration [25] likely discourages self-reports of injecting behavior; similarly, high levels of MSM stigmatization likely explain the complete absence of self-reported MSM among participants in this survey. Finally, HCV prevalence among participants reporting a history of IDU at some point in their lifetime may not reflect $\mathrm{HCV}$ prevalence among persons actively injecting drugs, due to changes in infection dynamics in injecting populations over time.

\section{Conclusions}

Georgia is working toward ambitious $\mathrm{HCV}$ elimination goals, aiming to screen and diagnose $90 \%$ of the estimated 150,000+ Georgians with chronic HCV infection, treat $95 \%$ of those identified, and reduce national prevalence of chronic HCV by $90 \%$ by 2020 [18]. This survey has provided nationally representative data to guide Georgia's comprehensive HCV elimination strategy, as well as baseline HCV prevalence to evaluate progress toward $\mathrm{HCV}$ elimination in the coming years. In addition, conducting the survey provided an important opportunity to strengthen Georgia's public health capacity and thereby enhance global health security

\section{Abbreviations \\ anti-HCV: antibodies to hepatitis C virus; AOR: adjusted odds ratio; \\ CDC: United States Centers for Disease Control and Prevention; \\ $\mathrm{Cl}$ : confidence interval; DAA: direct-acting antiviral; Geostat: Georgia National Statistics Office; HCV RNA: hepatitis C virus ribonucleic acid; HCV: hepatitis C virus; IDU: injection drug use; MSM: men who have sex with men; NCDC: Georgia National Center for Disease Control and Public Health}

\section{Acknowledgements}

The authors thank the Georgian residents who participated in this survey. In addition, we thank the following individuals for their training, laboratory, and field support: Kaeanne Parris, Monique Foster, Ashley Greiner, Sinead Isaacson, Jaclyn Perlman, Alexandra Tejada, Tracy Greene-Montfort, and Natasha Khudyakov, as well the CDC Field Epidemiology and Laboratory Training Program, and the interviewers, nurse-phlebotomists, and laboratorians who collected data and tested samples for this important initiative.

\section{Availability of data and material}

The datasets generated and analysed during this study are not publicly available due to privacy restrictions. Participants were informed during the consent process that the data they provide would be available only to the Georgian Ministry of Labour, Health, and Social Affairs and the US Centers for Disease Control and Prevention.

\section{Funding}

All funding for this study came from the US Centers for Disease Control and Prevention and the Georgia National Center for Disease Control. The findings and conclusions in this report are those of the authors and do not necessarily represent the official position of the Centers for Disease Control and Prevention.

\section{About this supplement}

This article has been published as part of BMC Public Health Volume 19 Supplement 3, 2019: 10th anniversary of the Centers for Disease Control and Prevention - Global Disease Detection program. The full contents of the supplement are available online at https://bmcpublichealth.biomedcentral. com/articles/supplements/volume-19-supplement-3.

\section{Authors' contributions}

FA, JM, AG, and PI conceived of the idea for this survey, and LH, SS, MA, GC, $\mathrm{CB}, \mathrm{GK}, \mathrm{SK}, \mathrm{JD}$, and FA contributed to its design. MA and GC oversaw laboratory procedures in Georgia, and NC and RS conducted laboratory analyses. JD oversaw the overall laboratory component, trained laboratory staff, and conducted laboratory quality assurance/quality control and confirmatory testing at CDC. LH, SS, AK, and MS trained interviewers and phlebotomists and oversaw the field work component. GK obtained data from Geostat to weight survey data. DB conducted follow-up data collection. $\mathrm{LH}$ and SR conducted statistical analyses, and LH, SR, CB, SH, and FA interpreted the results. $\mathrm{LH}$ and AK designed figures for the manuscript, and $\mathrm{LH}$ drafted the initial version of the manuscript. All authors have reviewed and approved the final manuscript.

\section{Ethics approval and consent to participate}

Ethical approval was obtained from the Georgia National Center for Disease Control and Public Health Institutional Review Board. This survey was determined to be a routine public health activity for public health surveillance by CDC's Human Subjects Research Office and therefore judged to not involve human subjects research. All participants signed a consent form in their preferred language; the form explained the purpose of the survey, provided contact information for the Principal Investigator, informed participants that they could decline to answer any questions they chose, and assured them that their participation would not influence health care services they receive through the Georgian government.

\section{Consent for publication}

Not applicable.

\section{Competing interests}

The authors declare that they have no competing interests.

\section{Publisher's Note}

Springer Nature remains neutral with regard to jurisdictional claims in published maps and institutional affiliations.

\section{Author details}

${ }^{1}$ Division of Viral Hepatitis, Centers for Disease Control and Prevention, Atlanta, GA, USA. ${ }^{2}$ National Center for Disease Control and Public Health, Tbilisi, Georgia. ${ }^{3}$ Division of Global Health Protection, Centers for Disease Control and Prevention, Atlanta, GA, USA. ${ }^{4}$ School of Public Health, Boston University, Boston, MA, USA. ${ }^{5}$ Global Disease Detection - South Caucasus Regional Center, Centers for Disease Control and Prevention, Tbilisi, Georgia.

Published: 10 May 2019

\section{References}

1. World Health Organization. Global Hepatitis Report 2017 [Available from: http://apps.who.int/iris/bitstream/10665/255016/1/9789241565455-eng. pdf?ua=1. Accessed 19 Jul 2017.]

2. Alter MJ. Epidemiology of hepatitis C virus infection. World J Gastroenterol. 2007;13(17):2436-41

3. Institute of Medicine. Hepatitis and liver cancer: a National Strategy for prevention and control of hepatitis B and C. Washington, DC; 2010.

4. Lauer GM, Walker BD. Hepatitis C virus infection. N Engl J Med. 2001;345(1): 41-52.

5. Lozano R, Naghavi M, Foreman K, Lim S, Shibuya K, Aboyans V, et al. Global and regional mortality from 235 causes of death for 20 age groups in 1990 
and 2010: a systematic analysis for the Global Burden of Disease Study 2010. Lancet. 2012;380(9859):2095-128.

6. World Health Organization. Hepatitis C Fact Sheet N 1642015 [Available from: http://www.who.int/mediacentre/factsheets/fs164/en/. Accessed 9 Feb 2016.]

7. Perz JF, Armstrong GL, Farrington LA, Hutin YJ, Bell BP. The contributions of hepatitis $B$ virus and hepatitis $C$ virus infections to cirrhosis and primary liver cancer worldwide. J Hepatol. 2006;45(4):529-38.

8. National Statistics Office of Georgia. Key Indicators 2015 [Available from: http:// www.geostat.ge/index.php?action=0\&lang=eng. Accessed 12 Feb 2016.]

9. Stvilia K, Tsertsvadze T, Sharvadze L, Aladashvili M, del Rio C, Kuniholm MH, et al. Prevalence of hepatitis C, HIV, and risk behaviors for blood-borne infections: a population-based survey of the adult population of T'bilisi, republic of Georgia. J Urban Health. 2006;83(2):289-98,

10. Mitruka K, Tsertsvadze T, Butsashvili M, Gamkrelidze A, Sabelashvili P, Adamia $\mathrm{E}$, et al. Launch of a Nationwide hepatitis C elimination program-Georgia, April 2015. MMWR Morb Mortal Wkly Rep. 2015;64(28):753-7.

11. Kish L. A procedure for objective respondent selection within the household. J Am Stat Assoc. 1949:44(247):380-7.

12. National Statistics Office of Georgia. Household Income 2015 [Available from: http://www.geostat.ge/index.php?action=page\&p_id=182\&lang=eng Accessed 5 Jul 2016.]

13. European Union HCV Policy Summit. HCV elimination manifesto: The beginning of the end Brussels, Belgium2016 [Available from: http://www. hcvbrusselssummit.eu/elimination-manifesto. Accessed 16 Sep 2016.]

14. Hepatitis Australia. Australia leads the world in curing hepatitis $\mathrm{C}$ as record numbers treated 2016 [Available from: http://www.hepatitisaustralia.com/ newsarticles/australia-leads-the-world-in-curing-hepatitis-c-as-recordnumbers-treated/28/7/2016. Accessed 16 Sep 2016.]

15. Oklahoma State Department of Health. Setting a path towards HCV elimination in the Cherokee Nation: government, academia, and pharma collaboration 2016 [Available from: http://www.hhs.gov/hepatitis/blog/2016/ 04/08/setting-path-towards-hcv-elimination-cherokee-nation-governmentacademia-and-pharma-collaboration. Accessed 16 Sep 2016.]

16. World Health Organization. Combating hepatitis B and C to reach elimination by 20302016 [Available from: http://www.who.int/hepatitis/ publications/hep-elimination-by-2030-brief/en/. Accessed 16/09/2016.

17. Gottfredsson M, Bergmann O, Tyrfingsson T, Runarsdottir V, Bjornsson E, Johannsson B, et al. TRAP HEP C - treatment as prevention for hepatitis $C$ in Iceland: a nationwide elimination program using direct acting antiviral agents. Stockholm: HIV \& Hepatitis Nordic Conference; 2017.

18. Gvinjilia L, Nasrullah M, Sergeenko D, Tsertsvadze T, Kamkamidze G, Butsashvili M, et al. National progress toward hepatitis C elimination Georgia, 2015-2016. MMWR Morb Mortal Wkly Rep. 2016;65(41):1132-5.

19. Edlin BR, Eckhardt BJ, Shu MA, Holmberg SD, Swan T. Toward a more accurate estimate of the prevalence of hepatitis $C$ in the United States. Hepatology. 2015;62(5):1353-63.

20. Harris A, Biddle J, Chokoshvili O, Turashvili K, Japaridze M, Burjanadze I, et al. A public health approach to hepatitis $C$ testing, care and treatment in the country of Georgia's corrections system. BMC Public Health. 2019;19(S3).

21. Sherriff $L C$, Mayon-White RT. A survey of hepatitis $C$ prevalence amongst the homeless community of Oxford. J Public Health Med. 2003;25(4):358-61.

22. Volf V, Marx D, Pliskova L, Sumegh L, Celko A. A survey of hepatitis B and C prevalence amongst the homeless community of Prague. Eur J Pub Health. 2008;18(1):44-7.

23. Kuniholm MH, Aladashvili M, Del Rio C, Stvilia K, Gabelia N, Chitale RA, et al. Not all injection drug users are created equal: heterogeneity of HIV, hepatitis C virus, and hepatitis B virus infection in Georgia. Subst Use Misuse. 2008:43(10):1424-37.

24. Nasrullah M, Sergeenko D, Gvinjilia L, Gamkrelidze A, Tsertsvadze T, Butsashvili $\mathrm{M}$, et al. The role of screening and treatment in National Progress toward Hepatitis C Elimination - Georgia, 2015-2016. MMWR Morb Mortal Wkly Rep. 2017;66(29):773-6.

25. Behavioral Surveillance Survey Report USAID. Characteristics of high-risk behaviors and knowledge of STI/HIV and prevalence of HIV, syphilis and hepatitis among injecting drug users in Tbilisi, Batumi, and Kutaisi, Georgia 2002-2006; 2006.

\section{Ready to submit your research? Choose BMC and benefit from:}

- fast, convenient online submission

- thorough peer review by experienced researchers in your field

- rapid publication on acceptance

- support for research data, including large and complex data types

- gold Open Access which fosters wider collaboration and increased citations

- maximum visibility for your research: over $100 \mathrm{M}$ website views per year

At BMC, research is always in progress.

Learn more biomedcentral.com/submissions 